\title{
Reversion of antibiotic resistance by inhibiting $m e c A$ in clinical methicillin-resistant Staphylococci by antisense phosphorothioate oligonucleotide
}

\author{
Jingru Meng ${ }^{1,4}$, Gonghao $\mathrm{He}^{2,4}$, Hui Wang ${ }^{3}$, Min $\mathrm{Jia}^{1}$, Xue Ma ${ }^{1}$, Fei Da ${ }^{1}$, Ning Wang ${ }^{1}$, Zheng Hou ${ }^{1}$, \\ Xiaoyan Xue ${ }^{1}$, Mingkai $\mathrm{Li}^{1}$, Ying Zhou ${ }^{1}$ and Xiaoxing Luo ${ }^{1}$
}

\begin{abstract}
Methicillin-resistant Staphylococci (MRS), methicillin-resistant Staphylococcus aureus (MRSA) and methicillin-resistant Staphylococcus epidermidis (MRSE) have become a challenging problem in nosocomial infections and are connected with high morbidity and mortality rates. This is due to the increasing incidence of resistance to virtually all $\beta$-lactams and a wide variety of antimicrobials. The spread of MRS severely limits therapeutic options and generates the need for novel antibiotics that are able to combat MRS infections. One method of inhibiting bacterial growth is by blocking the expression of conserved bacterial genes and provides potential new avenues for generating a new generation of antimicrobials. The mecA gene is highly conserved among Staphylococcal species, and this makes it an ideal target for antisense inhibition. We had identified a target sequence (854-871 nt) within the $m e c A$ mRNA coding region that is particularly sensitive to antisense inhibition. The anti-mecA PS-ODN04 oligonucleotide was encapsulated into an anionic liposome. MRSA01 and MRSE01 clinical strains treated with this antisense sequence became susceptible to existing $\beta$-lactam antibiotics, and their growth was inhibited by oxacillin in vitro and in vivo. PS-ODNO4 reduced the bacterial titers in the blood of mice infected with MRSAO1 and MRSE01 and significantly improved their survival rate. Our data offer a possible new strategy for treating MRS infections.
\end{abstract}

The Journal of Antibiotics (2015) 68, 158-164; doi:10.1038/ja.2014.132; published online 1 October 2014

\section{INTRODUCTION}

Staphylococcus is a Gram-positive bacteria that causes a wide range of infections in the blood, skin and wounds of its host. Staphylococci cause pathologies such as pneumonia and staphylococcal food poisoning in community and hospital settings by producing a toxin or causing invasion of tissues. ${ }^{1-3}$ Staphylococci are divided into two groups based on the presence or absence of the enzyme coagulase: coagulase-positive Staphylococcus (for example, Staphylococcus aureus) and coagulase-negative Staphylococcus (for example, Staphylococcus epidermidis). The high prevalence of antibiotic resistance, especially methicillin-resistant staphylococci (MRS), throughout the world has been a severe threat to public health. This increase in resistance is mainly due to the multi-resistant properties of Staphylococci. Since the first outbreaks of methicillin-resistant Staphylococcus aureus (MRSA) infections, the rates of MRSA and methicillin-resistant coagulasenegative Staphylococci (MRCNS) infections have increased worldwide $^{4-7}$ Therefore, developing a new arsenal of antimicrobial agents to combat the MRSA threat is an urgent priority.

One of the strategies used to develop this new set of antimicrobial agents is to use antisense oligonucleotides to inhibit resistance mechanisms at the genetic level. Antisense oligonucleotides are short (about 13-25 bases), single-stranded nucleotide molecules that bind complementary mRNA by sequence-specific base pairing to prevent translation. However, the use of antisense oligonucleotides is limited, as they have poor pharmacokinetics, are rapidly degraded by intracellular endonucleases and exonucleases, and possess weak binding to plasma proteins. Structural modifications can increase their nuclease stability and intrinsic affinity to complementary targets. Phosphorothioate oligodeoxynucleotides (PS-ODNs) are the most widely studied oligonucleotides. PS-ODNs are also capable of activating RNase $\mathrm{H}$ activity to degrade mRNA. ${ }^{8,9}$

To develop a new strategy to inhibit MRSA growth, we have chosen to block bacterial resistant genes using PS-ODNs. Our previous results have indicated that the inhibition of resistant gene expression by oligodeoxynucleotides strongly compromises the ability of these bacteria to evade conventional antimicrobial treatment. ${ }^{10-12} \mathrm{MRS}$, which is resistant to the antibiotic methicillin, expresses a modified penicillin-binding protein $2 \mathrm{a}$ ( $\mathrm{PBP} 2 \mathrm{a})$ encoded by the mecA gene. PBP2a is a novel and inducible PBP with a low affinity for $\beta$-lactam antibiotics, unlike intrinsic Staphylococci PBPs, which have a high affinity for $\beta$-lactam antibiotics. The $\beta$-lactam antibiotic is able to inhibit PBPs and kill Staphylococci, but MRS expressing PBP2a can

${ }^{1}$ Department of Pharmacology, School of Pharmacy, Fourth Military Medical University, Xi'an, China; ${ }^{2}$ Department of Pharmacy, Kunming General Hospital of Chengdu Military Region, Kunming, China and ${ }^{3}$ Jiuquan Satellite Launch Center, Jiuquan, China

${ }^{4}$ These authors contributed equally to this work.

Correspondence: Professor X Luo, Department of Pharmacology, The Fourth Military Medical University, 169 Changle West Rd., Xi'an 710032 , China.

E-mail: xxluo3@fmmu.edu.cn

Received 9 April 2014; revised 18 August 2014; accepted 27 August 2014; published online 1 October 2014 
continue to synthesize a cell wall by depending solely on the uninhibited activity of PBP2a. ${ }^{13-15}$

In the current study, we used a specific anti-mecA antisense PSODN encapsulated in an anionic liposome to inhibit the expression of mecA mRNA in clinically isolated pathogenic methicillin-resistant Staphylococcus epidermidis (MRSE) and (MRSA). The MRSE and MRSA strains that were used had varying degrees of resistance, and their response to the PS-ODN construct was evaluated both in vitro and in vivo. We examined whether anti-mecA PS-ODN could inhibit the expression of mecA and subsequently make MRS susceptible to $\beta$ lactam antibiotics. Furthermore, we identified the potential of mecA as a potent target for reversion of multidrug resistance in MRS.

\section{MATERIALS AND METHODS}

\section{Chemicals}

All the antibiotics used in this study were purchased from the National Institute for the Control of Pharmaceutical and Biological Products (Beijing, China). Polyethylenimine (PEI, $25 \mathrm{KDa}$ ) was purchased from Sigma-Aldrich (St Louis, MO, USA). Egg yolk phosphatidylcholine (EPC) was purchased from Xi'an Libang Pharmaceutical Co., Ltd. (Xi'an, China). N-(Carbonyl-methoxypolyethylene glycol 2000) -1,2-distearoylsn-glycerol-3-phosphoethanolamine ( $\mathrm{PEG}_{2000}$-DSPE) was purchased from Heli-Can Biotechnology Ltd. (Vancouver, BC, Canada). Dimyristoyl phosphatidylglycerol (DMPG) was purchased from Lipoid (Ludwigshafen, Germany). All the culture media was purchased from Beijing Land Bridge Technology (Beijing, China). All the chemicals and solvents used in this study were of analytical grade.

\section{Organisms}

Mu50 (ATCC700699) was purchased from MicroBiologics (Minnesota, MN, USA). The MRSA clinical strains MRSA01, MRSA02 and the MRSE clinical isolated strains MRSE01 and MRSE02 were isolated from cultures of sputum or catheter samples from patients in Xijing Hospital. The laboratory strain ATCC29213 (antibiotic sensitive, used for quality control) was obtained from the Chinese National Center for Surveillance of Antimicrobial Resistance (Beijing, China). The reverse transcription PCR (RT-PCR) analysis showed that Mu50, MRSA01, MRSA02, MRSE01 and MRSA02 expressed the mecA gene, whereas ATCC29213 did not express mecA (Supplementary Figure S1).

Five- to six-week-old, specific-pathogen-free male BALB/c mice weighing 18-20 $\mathrm{g}$ were used in this study. Standard laboratory chow and drinking water were provided ad libitum. A period of 3 days was allowed for the animals to acclimatize before experimentation took place. The experimental and animal care procedures were approved by the Animal Care and Use Committees of Fourth Military Medical University.

\section{Antisense oligonucleotides}

The sequence of the PS-ODNs used in this study is shown in Supplementary Table S3. The control-mismatched sequence (5'- cgactctacacgaacgtg-3), corresponding to the PS-ODNs, was randomly aligned with a sequence of the same number of bases. The PS-ODNs were synthesized by Aoke Biotechnology Limited-liability Company (Beijing, China) and were fully phosphorothioated.

\section{Homology analysis of target gene mecA and anti-mecA ODN optimization}

GeneBank was used to retrieve the sequence of a variety of the mecA genes from different kinds of Staphylococci, and the homology in the effective coding regions of the mecA gene was analyzed among these Staphylococci species (including Staphylococcus aureus, Staphylococcus epidermidis, Staphylococcus haemolyticus) using Blast.

We predicted the secondary structure of the mecA mRNA and analyzed the DNA:RNA and DNA:DNA paring parameters using RNA structure 4.6 software (Mathews Lab, Rochester, NY, USA) to determine the site with the best binding affinity. All of the PS-ODNs used were encapsulated into an anionic liposome (L-ODNs). The effective target site was verified by a modified MIC assay of L-ODNs in ATCC29213, MRSA01 and MRSE01.
Preparation of anionic liposomes encapsulated with nano-sized PSODN/PEI complexes

We encapsulated PS-ODNs into an anionic liposome to improve the efficiency of cell entry across stringent bacterial cell walls. To increase encapsulation efficiencies of anionic liposomes, PS-ODN and PEI were condensed into PSODN/PEI nanometer particles and then these nanometer complexes were enveloped into a liposome. Nanometer PS-ODN/PEI complexes and anionic liposomes were prepared as described previously. ${ }^{16}$ The encapsulation efficiency was determined as the percentage of the PS-ODN incorporated into the liposomes relative to the initial total amount of the PS-ODN according to the following equation: \% entrapment efficiency $=$ [amount of entrapped PS-ODN/ (amount of free PS-ODN+amount of entrapped PS-ODN) $] \times 100$. The encapsulation efficiency was found to be $78.6+3.2 \%$.

\section{Bacterial growth assay}

To determine the growth curve of MRSA01 and MRSE01 in the broth medium, $1.5 \times 10^{8} \mathrm{CFU}$ per $\mathrm{ml}$ cell dilution medium was mixed with liposome-encapsulated PBS, free PS-ODN04 $(18 \mu \mathrm{M})$, free PEI $(0.2 \mu \mathrm{M})$, liposome-encapsulated mismatched PS-ODN $(18 \mu \mathrm{M})$ or liposome-encapsulated PS-ODN04 $(0.7,2,6$ or $18 \mu \mathrm{m})$. Basically, $100 \mu \mathrm{l}$ mixtures containing $6 \mu \mathrm{g} \mathrm{ml}^{-1}$ oxacillin were added to the wells of a 96-well microtiter plate, and the culture was incubated at $35^{\circ} \mathrm{C}$ with agitation at 150 r.p.m. The growth rate of the cells was monitored by measuring the $\mathrm{OD}$ at $630 \mathrm{~nm}$ on a microplate reader (Bio-Rad Laboratories, Tokyo, Japan) at different time points.

To determine the kill curve for MRSA and MRSE, liposome-encapsulated PS-ODN04 were added to $1.0 \times 10^{6} \mathrm{CFU}$ per $\mathrm{ml}$ bacterial cultures to a final concentration of $0.7,2,6$ or $18 \mu \mathrm{M}$ with the addition of an equal volume of PBS as a control. Aliquots of each culture were collected at $0,3,6,9,12$ and $18 \mathrm{~h}$ and were diluted and inoculated on solid agar. The number of CFU was calculated from the number of colonies growing on the plates. Statistical data for each experiment were obtained from at least two independent assays performed in duplicate.

\section{Bacterial susceptibility testing}

MICs of oxacillin, flucloxacillin, piperacillin, cephalothin, cefoxitin and cefoperazone for the Mu50, MRSA and MRSE clinical strains were determined twice by the standard twofold broth dilution tube method according to the guidelines of the Clinical and Laboratory Standards Institute (CLSI). Quality control was assured by testing the control ATCC29213 strain in every experiment.

\section{RNA isolation and real-time RT-PCR reaction}

To confirm whether anti-mecA L-ODN04 could inhibit the expression of mecA mRNA, MRSA01 and MRSE01 were grown as described above and treated with liposome-encapsulated PBS, free PS-ODN04, free PEI, liposome-encapsulated mismatched PS-ODN or different concentrations of liposome-encapsulated PSODN04 for a total of $18 \mathrm{~h}$. The total RNA was extracted from the bacterial culture using Trizol reagent (Invitrogen, Carlsbad, CA, USA) according to the manufacturer's instructions. cDNA was prepared using random primers using PrimeScript RT reagent Kit (Takara biotechnology, Dalian, China) and analyzed with the SYBR Premix Ex Taq (Takara biotechnology) in a thermal cycler involved an initial denaturation step at $95^{\circ} \mathrm{C}$ for $2 \mathrm{~min}, 39$ cycles at $95^{\circ} \mathrm{C}$ for $30 \mathrm{~s}, 58^{\circ} \mathrm{C}$ for $30 \mathrm{~s}$ and $72^{\circ} \mathrm{C}$ for $30 \mathrm{~s}$. The melting curves of the PCR products were acquired by a stepwise increase of the temperature from 60 to $94^{\circ} \mathrm{C}$ (temperature transition $0.4^{\circ} \mathrm{C} \mathrm{s}^{-1}$ ) using the gene-specific oligonucleotide primers (Supplementary Table S1). gyrB was used as an internal control. Relative expression of mecA was calculated using the $2^{-\Delta \Delta \mathrm{Ct}}$ method and the gyrB gene for normalization.

\section{Therapeutic effects of anti-mecA L-ODN04 on infected animals} A respective infection was induced by the administration of $6.5 \times 10^{5} \mathrm{CFU}$ MRSA01 or $1 \times 10^{6} \mathrm{CFU}$ MRSE01inoculum in $0.4 \mathrm{ml}$ of Mueller-Hinton broth (with $5 \%$ mucin) intraperitoneally. Six mice were used in each group, and the bacterial suspension at $25 \mathrm{ml} \mathrm{kg}^{-1}$ of body weight was injected into each mouse. After bacterial challenge, the mice were randomly chosen to receive $100 \mathrm{mg} \mathrm{kg}^{-1}$ 
oxacillin (twice daily for 7 days), 5 and $10 \mathrm{mg} \mathrm{kg}^{-1} \mathrm{~L}$-ODN04 (once daily for 3 days) and $100 \mathrm{mg} \mathrm{kg}^{-1}$ oxacillin (twice daily for 7 days) or the same amount of isotonic solution $30 \mathrm{~min}$ after infection. The injections were given intravenously via the tail vein. Blood samples were obtained from the tail vein by aseptic percutaneous puncture $24 \mathrm{~h}$ after bacterial challenge. The blood samples were cultured on Mueller-Hinton agar plates, and the CFU in the blood samples were calculated. The survival of six mice in each group was monitored every $6 \mathrm{~h}$ for 7 days post infection, and the cumulative percentage of mice that had survived the study was determined.

\section{Statistical analysis}

The results were expressed as the mean \pm s.d. The resultant data were analyzed by using a one-way analysis of variance, followed by the Student - NewmanKeuls test. The animal survival curves were tested by Kaplan - Meier analyses with the probability value of $P<0.05$, considered indicative of statistical significance.

\section{RESULTS}

Homology analysis of target gene mecA and the determination of mecA target site

The mecA gene is well conserved across species of Staphylococci, and the mecA gene encoding PBP2a has a high homology between species. Sequence alignment of Staphyloccus mecA by Blast showed 100\% gene identity in S. aureus, S. epidermidis and other Staphyloccus (that is, Staphylococcus haemolyticus, Staphylococcus hominis, Staphylococcus saprophyticus, Staphylococcus cohnii, Staphylococcus capitis and Staphylococcus pseudintermedius; Supplementary Table S2). Therefore, we chose the mecA gene as the target gene to reverse the resistance of MRS in our study.

Secondary structure of the mecA mRNA was predicted (Supplementary Figure S2), and the DNA:RNA and the DNA:DNA binding parameters were calculated (Supplementary Table S3) by RNA structure 4.6 software. The combined data showed that mecA mRNA has generous conceivable subregions for antisense targeting. Some investigators have observed that the translation start codon region of mRNA is the most sensitive to antisense. ${ }^{17,18}$ However, we have found there were no subregions that were suitable targets for antisense in the translation start codon region of mecA mRNA. Therefore, we selected six plausible target sites in other coding regions for the target regions of the 18-mer oligodeoxynucleotides (ODNs) according to the binding parameters including overall $\Delta \mathrm{G}$, Duplex $\Delta \mathrm{G}$, Tm, Intraoligo $\Delta \mathrm{G}$, Interoligo $\Delta \mathrm{G}$ and $\mathrm{GC} \%$ content (Supplementary Figure S2 and Supplementary Table S3). ${ }^{19,20}$ All of the six mRNA regions are devoid of obvious stable secondary structures; thus, they are theoretically accessible to complementary ODNs. Corresponding to the six target sites, we designed six ODNs, which were complementary to the mecA mRNA nucleotide (nt) encoding regions 182-199 (ODN1), 328-345 (ODN2), 375-392 (ODN3), 854-871 (ODN4), 1170-1187 (ODN5) and 1673-1690 (ODN6).

\section{Optimization of anti-mecA PS-ODNs}

All PS-ODNs used are encapsulated into anionic liposomes (L-ODNs). The identification of the sensitive region of gene mecA and optimization of anti-mecA PS-ODNs for efficacious gene-specific antisense inhibition were verified by MIC assay of L-ODNs in strains ATCC29213, MRSA01 and MRSE01 (Table 1). All L-ODNs didn't affect the MIC value of oxacillin in strain ATCC29213. L-ODN01 was inactive at the highest concentration tested $(18 \mu \mathrm{M})$ in strain MRSE01, whereas L-ODN01 slightly decreased the MIC value of oxacillin in strain MRSA01. Meanwhile L-ODN03 also slightly decreased the MIC value of oxacillin in strains MRSA01 and MRSE01. It is important to note that L-ODN02, L-ODN04, L-ODN05 and L-ODN06 all could
Table 1 MICs of oxacillin for quality control and clinically isolated strain MRSA01 and MRSE01 in the presence or absence of anti$m e c A$ PS-ODNs encapsulated into liposomes in $\mathrm{M}-\mathrm{H}$ broth culture

\begin{tabular}{|c|c|c|c|c|c|c|}
\hline \multirow[b]{3}{*}{ PS-ODNs $(18 \mu \mathrm{M})$} & \multicolumn{6}{|c|}{ MICs of oxacillin $\left(\mu g m l^{-1}\right)^{a}$} \\
\hline & \multicolumn{2}{|c|}{ ATCC29213 } & \multicolumn{2}{|c|}{ MRSAO1 } & \multicolumn{2}{|c|}{ MRSEO1 } \\
\hline & Non-ODN & $O D N$ & Non-ODN & $O D N$ & Non-ODN & $O D N$ \\
\hline L-ODN01 & 0.5 & 0.5 & 1024 & 512 & 512 & 512 \\
\hline L-ODN02 & 0.5 & 0.5 & 1024 & 4 & 512 & 0.5 \\
\hline L-ODN03 & 0.5 & 0.5 & 1024 & 128 & 512 & 256 \\
\hline L-ODNO4 & 0.5 & 0.5 & 1024 & 2 & 512 & 0.13 \\
\hline L-ODN05 & 0.5 & 0.5 & 1024 & 2 & 512 & 0.25 \\
\hline L-ODN06 & 0.5 & 0.5 & 1024 & 4 & 512 & 0.25 \\
\hline
\end{tabular}

Abbreviation: L-ODNs, liposome-encapsulated PS-ODNs.

${ }^{a}$ Classification of Staphylococcus aureus strains by MIC of oxacillin, sensitive, MIC $\leqslant 2 \mu \mathrm{g} \mathrm{ml}^{-1}$; resistant, $\mathrm{MIC} \geqslant 4 \mathrm{\mu g} \mathrm{ml}^{-1}$.

Classification of Staphylococcus epidermidis strains by MIC of oxacillin, sensitive,

$\mathrm{MIC} \leqslant 0.25 \mu \mathrm{g} \mathrm{ml}^{-1}$; resistant, MIC $\geqslant 0.5 \mu \mathrm{g} \mathrm{m}^{-1}$. The experiment was repeated three times.

significantly decrease the MIC value of oxacillin in strains MRSA01 and MRSE01 (Table 1). As a result, we hypothesized that the translation start or non-start coding region of the target gene could be used as an effective target site. The sequence of the most active ODNs L-ODN04 was 5'-AACCATTTTTCCCTGAGC-3', which is complementary in sequence to nucleotide $854-871$ in the coding region of the mecA mRNA. The control-mismatched sequence of this antisense L-ODN04 is 5'-TCACCTGTTCACTGATAC-3', which was randomly aligned (L-misODN).

\section{Reversal of antibiotic resistance of MRSA and MRSE strains by encapsulated anti-mecA PS-ODN04 and inhibition of MRSA and MRSE growth by oxacillin}

The experiments were performed to determine the growth rate of MRSA01 and MRSE01 in liquid medium containing $6 \mu \mathrm{g} \mathrm{ml}^{-1}$ of oxacillin (Figure 1). In the MRSA01 and MRSE01 cultures, we observed a time- and concentration-dependent inhibition of bacterial growth by oxacillin in the L-ODN04-treated cells. The growth of MRSA01 and MRSE01 was inhibited by L-ODN04 in a concentrationdependent manner compared with cells grown in the control group (Figures 1a and b). However, the growth of MRSA01 and MRSE01 in liquid medium containing $6 \mu \mathrm{g} \mathrm{ml}^{-1}$ of oxacillin was not influenced by the treatment with liposome-encapsulated PBS, liposome-encapsulated mismatched PS-ODN $(18 \mu \mathrm{M})$, free PEI $(0.2 \mu \mathrm{M})$ or free PS-ODN04 (18 $\mu \mathrm{m}$; Supplementary Figure S3). At the same time, the growth of ATCC29213, which did not express mecA, was not influenced by antimecA encapsulated L-ODN04 $(18 \mu \mathrm{M})$. However, the growth of ATCC29213 was inhibited by $6 \mu \mathrm{g} \mathrm{ml}^{-1}$ oxacillin (Figure 1c). These results suggested that ODN04 could target the mecA gene and reversed the resistance of MRS to oxacillin.

Studies of the kill curves showed that L-ODN04 combining with oxacillin reduced the bacterial population from $10^{6}$ to about 0 in a time- and concentration-dependent manner. Oxacillin displayed a potent growth inhibitory effect on MRSA01 and MRSE01 when the bacteria were treated with encapsulated L-ODN04 at 0.7, 2, 6 and $18 \mu \mathrm{M}$. However, the growth of the bacteria was not influenced when they were treated with only $6 \mu \mathrm{g} \mathrm{ml}^{-1}$ oxacillin (Figure 2 ). The results indicate that anti-mecA L-ODN04 can restore the susceptibility of MRS to oxacillin such that oxacillin is able to inhibit the growth of MRSA and MRSE. 
a

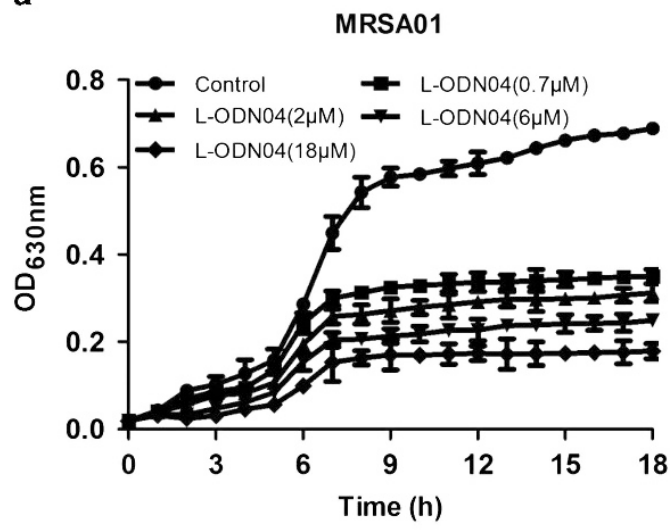

b

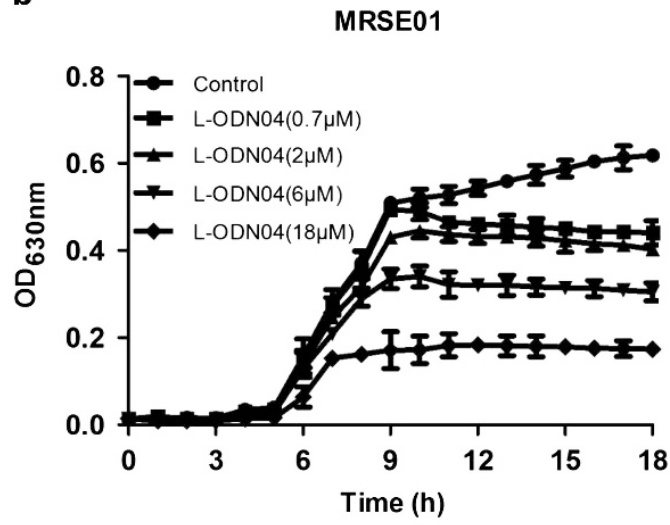

C

ATCC29213

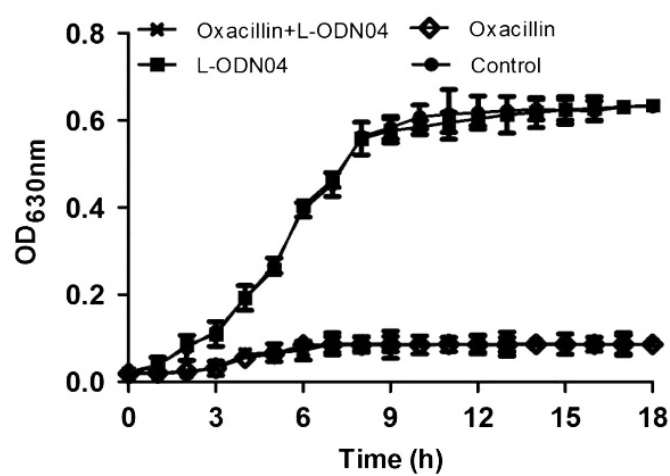

Figure 1 Effect of anti-mecA PS-ODNO4 encapsulated into nanoparticle liposomes on the growth of the MRSA clinical strain MRSAO1 (a), MRSE clinical strain MRSEO1 (b) and MSSA strain ATCC29213 in liquid cultures. The cells were cultured in liquid medium. Growth of the strains in the different groups was monitored at $\mathrm{OD}_{630}$. (a, b) Bacteria were treated with encapsulated PS-ODNO4 at a final concentration of $0.7,2,6$ or $18 \mu \mathrm{m}$ combined with $6 \mu \mathrm{g} \mathrm{m}^{-1}$ oxacillin as indicated on the graph, with the addition of an equal volume of free PBS as a control. (c) Bacteria were treated with PBS (control), $6 \mu \mathrm{g} \mathrm{ml}^{-1}$ oxacillin, $18 \mu \mathrm{m}$ encapsulated PSODNO4 and encapsulated PS-ODNO4 combined with $6 \mu \mathrm{g} \mathrm{ml}{ }^{-1}$ oxacillin. The data were shown as mean \pm s.d. of 10 samples.

Restoration of antibiotic susceptibility of MRSA and MRSE strains to clinical commonly used antibiotics

Furthermore, we observed that there was a change of MICs of the six commonly used clinical antibiotics against ATCC29213, Mu50, MRSA

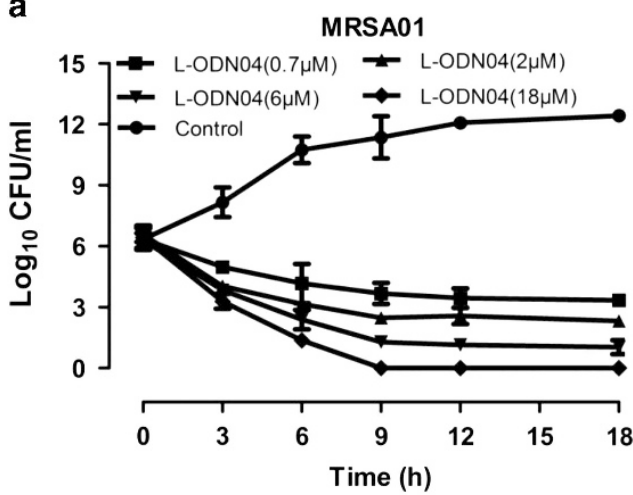

b

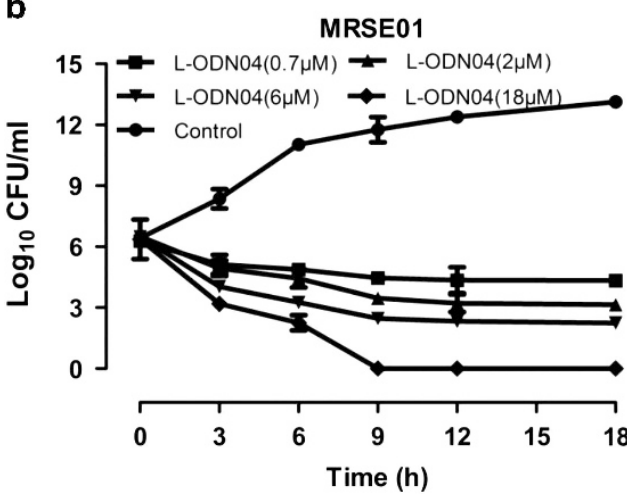

Figure 2 Kill curves of anti-mecA PS-ODNO4 encapsulated into nanoparticle liposomes combined with oxacillin against MRSAO1 and MRSEO1. Encapsulated PS-ODNO4 at a final concentration of $0.7,2,6$ or $18 \mu \mathrm{m}$ combined with $6 \mu \mathrm{g} \mathrm{ml}^{-1}$ oxacillin were added to cell cultures containing the MRSA clinical strain MRSAO1 (a) and MRSE clinical strain MRSEO1 (b), with the addition of an equal volume of diluent as a control. Aliquots of each culture were collected at $0,3,6,9,12$ and $18 \mathrm{~h}$ and were then diluted and inoculated on solid agar. The number of CFUs was calculated from the number of colonies growing on plates. The data were shown as mean \pm s.d. of 10 samples.

and MRSE clinical isolated strains in the presence or absence of L-ODN04 at $18 \mu \mathrm{M}$. Mu50, MRSA01, MRSA02, MRSE01 and MRSE02 all showed a strong resistance to oxacillin, flucloxacillin, cephalothin, cefoxitin and cefoperazone. The encapsulated PS-ODN04 (18 $\mu \mathrm{M})$ treatment could reduce the MICs of all of the six antibiotics from 161024 to $0.13-64 \mu_{\mathrm{g} \mathrm{ml}}{ }^{-1}$, respectively (Table 2). However, ATCC29213 was a control MSSA strain and was susceptible to oxacillin, flucloxacillin, cephalothin, cefoxitin and cefoperazone. Meanwhile, encapsulated anti-mecA L-ODN04 did not influence the MICs of all of the six antibiotics for ATCC29213. The results revealed that the encapsulated anti-mecA PS-ODN04 lowered the MICs of oxacillin, flucloxacillin, piperacillin, cephalothin, cefoxitin and cefoperazone against five MRSA and MRSE strains, some of which reached the sensitive margin values for these antibiotics to $S$. aureus and S. epidermidis strains on the basis of the interpretive criteria recommended by the CLSI (Table 2).

Real-time quantified assays for mecA expression

Next, we determined whether downregulation of mecA by the addition of anti-mecA L-ODN04 was correlated with the restoration of antibiotic susceptibility in MRSA and MRSE. Using the $2^{-\Delta \Delta C t}$ realtime PCR method, the change in gene expression of mecA mRNA in 
Table 2 MICs of antibiotics for ATCC29213, Mu50, MRSA clinically isolated strains (MRSA01 and MRSA02), and MRSE clinical isolated strains (MRSE01 and MRSE02) in the presence or absence of $18 \mu \mathrm{m}$ anti-mecA PS-ODN04 encapsulated into nanoparticle liposomes in M-H broth culture

MICs of antibiotics $\left(\mu g \mathrm{ml}^{-1}\right)^{\mathrm{a}}$

\begin{tabular}{|c|c|c|c|c|c|c|c|c|c|c|c|c|}
\hline Antibiotics & Non-ODN & $O D N$ & Non-ODN & $O D N$ & Non-ODN & $O D N$ & Non-ODN & $O D N$ & Non-ODN & $O D N$ & Non-ODN & $O D N$ \\
\hline FLU & 0.5 & 0.5 & 512 & 4 & 1024 & 2 & 512 & 8 & 256 & 1 & 512 & 4 \\
\hline PIP & 2 & 2 & - & - & - & - & - & - & 16 & 4 & 32 & 4 \\
\hline CEP & 4 & 4 & 1024 & 32 & 512 & 8 & 256 & 16 & 512 & 4 & 256 & 32 \\
\hline FOX & 2 & 2 & 1024 & 64 & 512 & 4 & 256 & 32 & 32 & 0.25 & 128 & 16 \\
\hline
\end{tabular}

Abbreviations: CEP, cephalothin; CFP, cefoperazone; FLU, flucloxacillin; FOX, cefoxitin; OXA, oxacillin; PIP, piperacillin.

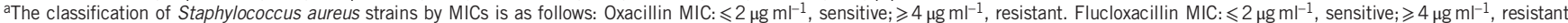
Cephalothin and Cefoxitin MICs: $\leqslant 8 \mu \mathrm{g} \mathrm{ml} l^{-1}$, sensitive; $16 \mu \mathrm{g} \mathrm{ml}^{-1}$, intermediate; $\geqslant 32 \mu \mathrm{g} \mathrm{ml} l^{-1}$, resistant. Cefoperazone MIC: $\leqslant 16 \mu \mathrm{g} \mathrm{ml} l^{-1}$, sensitive; $32 \mu \mathrm{g} \mathrm{ml} l^{-1}$, intermediate; $\geqslant 64 \mu \mathrm{g} \mathrm{ml} l^{-1}$, resistant. The classification of Staphylococcus epidermidis strains by MICs is as follows: Oxacillin MIC: $\leqslant 0.25 \mu \mathrm{g} \mathrm{ml}^{-1}$, sensitive; $\geqslant 0.5 \mu \mathrm{g} \mathrm{ml} l^{-1}$, resistant. Cephalothin MICs: $\leqslant 8 \mu \mathrm{g} \mathrm{ml}-1$, sensitive; $16 \mu \mathrm{g} \mathrm{ml}-1$, intermediate; $\geqslant 32 \mu \mathrm{g} \mathrm{ml}^{-1}$, resistant. Cefoperazone MIC: $\leqslant 16 \mu \mathrm{g} \mathrm{ml}^{-1}$, sensitive; $32 \mu \mathrm{g} \mathrm{ml}^{-1}$, intermediate; $\geqslant 64 \mu \mathrm{g} \mathrm{ml}{ }^{-1}$, resistant. The experiment was repeated three times.

MRSA01 and MRSE01 was normalized to gyrB mRNA. Compared with the control group treated with PBS, the relative expression of $m e c A$ by MRSA01 treated with anti-mecA L-ODN04 at different concentrations was decreased in a concentration-dependent manner to $49,29,18$ and $10 \%$ of control values (Figure 3a, Supplementary Table S4). At the same time, different concentrations of L-ODN04 decreased the expression of mecA of MRSE01 to 32, 24, 17 and 10\% of the control values (Figure 3b, Supplementary Table S5). The relative expression of mecA in the MRSA01 and MRSE01 cells treated with free PS-ODN04 was 82 and 68\%, respectively, compared with the control group. Moreover, no detectable changes in mecA expression were observed in the liposome-encapsulated PBS, liposome-encapsulated mismatched PS-ODN and free PEI-treated cells. This result demonstrates that the encapsulated PS-ODN04 not only enters the bacterial cells, but also interacts with the mecA mRNA, and then inhibits the expression of mecA mRNA.

\section{In vivo protective effects of the encapsulated PS-ODN04}

To test whether L-ODN04 could provide a survival benefit in vivo, whole animal infection experiments were performed using MRSA01 or MRSE01. All of the infected mice in the control group (treated with isotonic solution) and the $100-\mathrm{mg} \mathrm{kg}^{-1}$ oxacillin-treated group have died within 3 days. The administration of each dose of L-ODN04 (5 and $10 \mathrm{mg} \mathrm{kg}^{-1}$ ) in either MRSA01 or MRSE01 infected mice significantly improved the animals' survival rate from $0 \%$ for the control mice to 16.7 and $33.3 \%$ for the MRSA01-infected mice (Figure $4 \mathrm{a}$ ) and from $0 \%$ for the control groups to 33.3 and $50 \%$ in MRSE01 group (Figure 4b). Furthermore, the increased survival rate was associated with reductions in the blood bacterial titers of mice inoculated with MRSA01 (from $1.95 \times 10^{11} \mathrm{CFU}$ per $\mathrm{ml}$ to $1.99 \times 10^{6}$ and $1.78 \times 10^{4} \mathrm{CFU}$ per $\mathrm{ml}$ ) and MRSE01 (from $2.51 \times 10^{11} \mathrm{CFU}$ per $\mathrm{ml}$ to $1.62 \times 10^{5}$ and $6.31 \times 10^{2} \mathrm{CFU}$ per $\mathrm{ml}$ ) (Figures $4 \mathrm{c}$ and $\mathrm{d}$ ). Treatment with oxacillin alone did not show any protective effects, either by decreasing the mortality rate of infected mice or by altering the CFU counts in blood samples from the septic mice.

\section{DISCUSSION}

Antibiotic multi-resistant Staphylococci represent a challenging problem, and MRSA and MRCNS are the most common Staphylococci isolated from clinical samples. ${ }^{21-23}$ Resistant Staphylococci, especially MRCNS, have become an increasing problem as a nosocomial infection that is connected with high morbidity and mortality
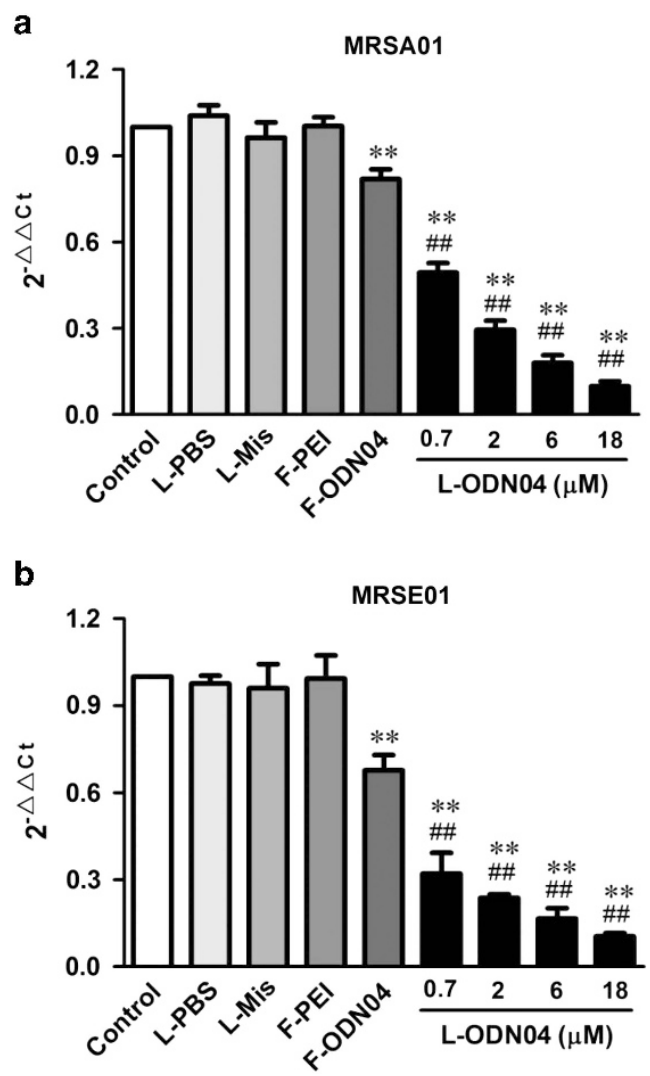

Figure 3 Relative mRNA expression of mecA of MRSA01 (a) and MRSE01 (b) as detected by real-time PCR. Bacteria were treated with free PBS (control), encapsulated PBS liposomes (L-PBS), free PEI (F-PEI, $0.2 \mu \mathrm{m}$ ),

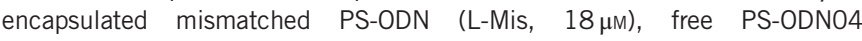
(F-ODN04, $18 \mu \mathrm{m}$ ), or encapsulated PS-ODN04 at different concentrations (L-ODN04). The data were fit using least-squares linear regression analysis. $C_{t}$ refers to cycle numbers when the fluorescence reaches fluorescence threshold. $\Delta \Delta C_{\mathrm{t}}=\left(C_{\mathrm{t} \cdot \mathrm{mec} A}-C_{\mathrm{t} \cdot \text { gyrB }}\right)_{\text {treatment }}-\left(C_{\mathrm{t} \cdot \mathrm{mec} A}-C_{\mathrm{t} \cdot \text { gyrB }}\right)_{\text {control. }}$ (mean \pm s.d., $n=3$ ). ${ }^{* *} P<0.01$ vs control, ${ }^{* \#} P<0.01$ vs free PS-ODN04.

rates. $22,24,25$ There is an increase in the incidence of microbes that display resistance to the first line antibiotics (such as $\beta$-lactams) and that have a reduced susceptibility to newer agents including vancomycin, linezolid and daptomycin. This increase in resistant bacteria 
a

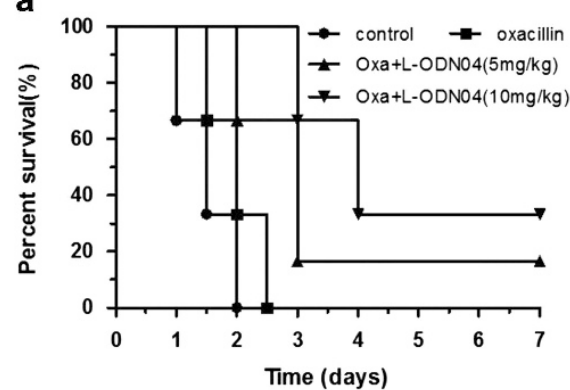

C

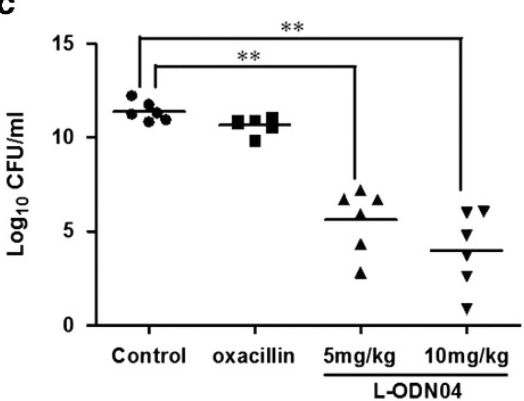

b

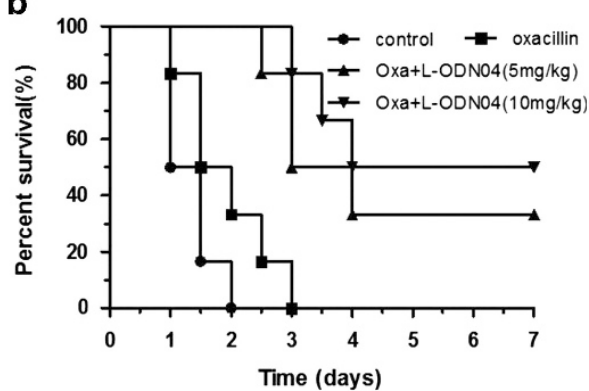

d

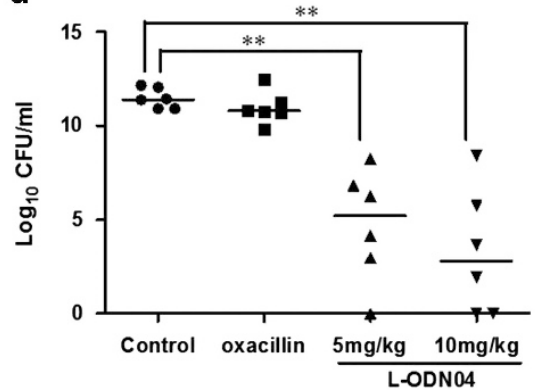

Figure 4 Effects of encapsulated PS-ODNO4 in a mouse model of MRSA/MRSE-induced sepsis. BALB/c mice were intraperitoneally inoculated with MRSA01 or MRSE01. Survival plot of BALB/C mice inoculated by intraperitonial injection with MRSAO1 $\left(6.5 \times 10^{5} \mathrm{CFU}\right)$ (a) or MRSEO1 ( $\left.1 \times 10^{6} \mathrm{CFU}\right)$ was generated (b). The mice were treated with $100 \mathrm{mg} \mathrm{kg}^{-1}$ oxacillin (twice daily for 7 days), 5 and $10 \mathrm{mg} \mathrm{kg}^{-1}$ liposomes encapsulated anti-mecA PS-ODNO4 (once daily for 3 days) and $100 \mathrm{mg} \mathrm{kg}^{-1}$ oxacillin (twice daily for 7 days) (oxa+L-ODNO4) or the same amount of isotonic solution. Colonization of MRSA01 (c) and MRSE01 (d) inoculum in the blood cultures of BALB/c mice treated with anti-mecA PS-ODN04 or isotonic solution. $n=6,{ }^{* *} P<0.01$ vs control.

has exceeded our ability to develop new antimicrobials and represents a serious concern for clinicians. ${ }^{26,27}$ It has been particularly challenging to discover and develop new antibiotics to methicillin-resistant and multidrug- resistant Staphylococci. Therefore, there is an urgent need for new strategies to combat MRS infections with mechanisms radically different from existing strategies.

Antisense-based antibiotics are a new and revolutionary approach for the targeting of these resistant microbes. Interrupting expression of resistance genes using antisense oligodeoxynucleotide could restore the antibiotic susceptibility of these bacteria and extend the lifespan of existing antibiotics. This would allow clinically proven therapies to be reutilized and be more valuable economically. ${ }^{28}$ For this reason, our and other's studies explored the use of antisense antibiotics to target antibiotic resistance genes, especially targeting bacterial species with the highest incidence of resistance, for example, $a a c\left(6^{\prime}\right)-I b$ and $a c r B$ in Escherichia coli, ${ }^{10,29}$ oprM in Pseudomonas aeruginosa ${ }^{12}$ and mecA in Staphylococcus aureus. ${ }^{16}$ Moreover, antisense antibacterial strategies have been proven to be more effective, practical and economical in treating multiple-resistant bacteria when they are coadministered with existing antibiotics.

Staphylococcal cassette chromosome mec is a genetic mobile element that carries the mecA gene and mediates methicillin resistance in Staphylococci. The results from our and other's research have shown that mecA is highly conserved among Staphylococcal species, including S. aureus, S. epidermidis, S. haemolyticus and S. saprophyticus, etc. (Supplementary Table S3). ${ }^{30,31}$ Previously, we found that antisense oligodeoxynucleotide targeted to mecA could significantly restore the susceptibility of WHO-2, a MRSA strain obtained from Chinese National Center for Surveillance of Antimicrobial Resistance, to existing $\beta$-lactam antibiotics, ${ }^{16}$ but we did not evaluate whether mecA could be an efficient target for antisense agents in clinical pathogenic
Staphylococci. Therefore, in this study we chose the mecA gene of resistant Staphylococci as the target for the antisense strategy.

In our study, the anti-mecA PS-ODN04 encapsulated into anionic liposomes significantly inhibited expression of mecA mRNA of MRSA01 and MRSE01 in a concentration-dependent manner. Furthermore, oxacillin was bacteriocidal for MRSA01 and MRSE01 when coadministered with anti-mecA L-ODN04 in vitro and in vivo. In summary, our study has indicated that inhibiting expression of mecA mRNA by antisense antibiotics could eventually reverse MRS resistance, and this may be a promising strategy for combating MRS infections.

\section{CONFLICT OF INTEREST}

The authors declare no conflict of interest.

\section{ACKNOWLEDGEMENTS}

This research was supported by grants from the National Natural Science Foundation of China (No. 30901813) and Natural Science Foundation of Shaanxi Province (No. 2012JM4019).

1 Fry, D. E. The continued challenge of Staphylococcus aureus in the surgical patient. Am Surg. 79, 1-10 (2013)

2 Marchant E. A., Boyce G. K., Sadarangani M. \& Lavoie P. M. Neonatal sepsis due to coagulase-negative staphylococci. Clin. Dev. Immunol. 2013, 586076 (2013).

3 Kalaivani V., Hiremath B. V. \& Indumathi V. A. Necrotising soft tissue infection-risk factors for mortality. J. Clin. Diagn. Res. 7, 1662-1665 (2013).

4 Benner E. J. \& Kayser F. H. Growing clinical significance of methcillin-resistant Staphylococcus aureus. Lancet 2, 741-744 (1968).

5 Lo, W. T. et al. Changing trends in antimicrobial resistance of major bacteria pathogens, 1985-2005: a study from a medical center in northern Taiwan. J. Microbiol. Immunol. Infect. 44, 131-138 (2011). 
6 Mertz, D. et al. Eradication of an epidemic methicillin-resistant Staphylococcus aureus (MRSA) from a geriatric university hospital: evidence from a 10-year follow-up. Eur. J. Clin. Microbiol. Infect. Dis. 29, 987-993 (2010).

7 Pereira, C. A. et al. Nosocomial bloodstream infections in Brazilian pediatric patients: microbiology, epidemiology, and clinical features. PLoS ONE 8, e68144 (2013).

8 Dias N. \& Stein C. A. Antisense oligonucleotides: basic concepts and mechanisms. Mol. Cancer Ther. 1, 347-355 (2002).

9 Bai, H. et al. Antisense antibiotics: a brief review of novel target discovery and delivery. Curr. Drug Discov. Technol. 7, 76-85 (2010).

$10 \mathrm{Meng}$, J. et al. Restoration of antibiotic susceptibility in fluoroquinolone-resistant Escherichia coli by targeting acrB with antisense phosphorothioate oligonucleotide encapsulated in novel anion liposome. J. Antibiot. (Tokyo) 65, 129-134 (2012).

11 Meng, J. et al. Restoration of oxacillin susceptibility in methicillin-resistant Staphylococcus aureus by blocking the MecR1-mediated signaling pathway. J. Chemother. 18, 360-365 (2006).

12 Wang, $\mathrm{H}$. et al. oprM as a new target for reversion of multidrug resistance in Pseudomonas aeruginosa by antisense phosphorothioate oligodeoxynucleotides. FEMS Immunol. Med. Microbiol. 60, 275-282 (2010).

13 Anstead G. M., Quinones-Nazario G. \& Lewis J. S. 2nd. Treatment of infections caused by resistant Staphylococcus aureus. Methods Mol. Biol. 391, 227-258 (2007).

14 Fuda C., Suvorov M., Vakulenko S. B. \& Mobashery S. The basis for resistance to betalactam antibiotics by penicillin-binding protein $2 a$ of methicillin-resistant Staphylococcus aureus. J. Biol. Chem. 279, 40802-40806 (2004).

15 Ghuysen, J. M. Molecular structures of penicillin-binding proteins and beta-lactamases. Trends Microbiol. 2, 372-380 (1994).

16 Meng, J. et al. Novel anion liposome-encapsulated antisense oligonucleotide restores susceptibility of methicillin-resistant Staphylococcus aureus and rescues mice from lethal sepsis by targeting mecA. Antimicrob. Agents Chemother. 53, 2871-2878 (2009).

$17 \mathrm{Bai}, \mathrm{H}$. et al. Antisense inhibition of gene expression and growth in gram-negative bacteria by cell-penetrating peptide conjugates of peptide nucleic acids targeted to rpoD gene. Biomaterials 33, 659-667 (2012).

18 Dryselius R., Aswasti S. K., Rajarao G. K., Nielsen P. E. \& Good L. The translation start codon region is sensitive to antisense PNA inhibition in Escherichia coli. Oligonucleotides 13, 427-433 (2003).
19 Cairns, M. J., Hopkins, T. M., Witherington, C., Wang, L. \& Sun, L. Q. Target site selection for an RNA-cleaving catalytic DNA. Nat. Biotechnol. 17, 480-486 (1999).

20 Matveeva, O. V. et al. Thermodynamic criteria for high hit rate antisense oligonucleotide design. Nucleic Acids Res. 31, 4989-4994 (2003).

21 Mshangila, B. et al. External ocular surface bacterial isolates and their antimicrobia susceptibility patterns among pre-operative cataract patients at Mulago National Hospital in Kampala, Uganda. BMC Ophthalmol. 13, 71 (2013).

22 Tak V., Mathur P., Lalwani S. \& Misra M. C. Staphylococcal blood stream infections: epidemiology, resistance pattern and outcome at a level 1 Indian trauma care center. J. Lab. Physicians 5, 46-50 (2013).

23 Titecat, M. et al. Bacterial epidemiology of osteoarticular infections in a referent center: 10-year study. Orthop. Traumatol. Surg. Res. 99, 653-658 (2013).

24 Brzychczy-Wloch, M. et al. Prevalence of antibiotic resistance in multi-drug resistant coagulase-negative staphylococci isolated from invasive infection in very low birth weight neonates in two polish NICUs. Ann. Clin. Microbiol. Antimicrob. 12, 41 (2013).

25 May L., Klein E. Y., Rothman R. E. \& Laxminarayan R. Trends in antibiotic resistance in coagulase negative Staphylococci, United States, 1999-2012. Antimicrob. Agents Chemother. 58, 1404-1409 (2014).

26 Rybak J. M., Barber K. E. \& Rybak M. J. Current and prospective treatments for multidrug-resistant gram-positive infections. Expert Opin. Pharmacother. 14, 1919-1932 (2013).

27 Kern, W. V. Management of Staphylococcus aureus bacteremia and endocarditis: progresses and challenges. Curr. Opin. Infect. Dis. 23, 346-358 (2010).

28 Woodford N. \& Wareham D. W. Tackling antibiotic resistance: a dose of common antisense? J. Antimicrob. Chemother. 63, 225-229 (2009).

29 Sarno R., Ha H., Weinsetel N. \& Tolmasky M. E. Inhibition of aminoglycoside 6'-Nacetyltransferase type Ib-mediated amikacin resistance by antisense oligodeoxynucleotides. Antimicrob. Agents Chemother. 47, 3296-3304 (2003).

30 Archer G. L., Niemeyer D. M., Thanassi J. A. \& Pucci M. J. Dissemination among staphylococci of DNA sequences associated with methicillin resistance. Antimicrob. Agents Chemother. 38, 447-454 (1994).

31 Ubukata K., Nonoguchi R., Song M. D., Matsuhashi M. \& Konno M. Homology of mecA gene in methicillin-resistant Staphylococcus haemolyticus and Staphylococcus simulans to that of Staphylococcus aureus. Antimicrob. Agents Chemother. 34 170-172 (1990).

Supplementary Information accompanies the paper on The Journal of Antibiotics website (http://www.nature.com/ja) 